\title{
Spatial structure of drift-wave turbulence and transport in a stellarator
}

\author{
G Birkenmeier ${ }^{1,2} \ddagger$, M Ramisch ${ }^{1}$, G Fuchert ${ }^{1}$, A Köhn ${ }^{1}$, B Nold ${ }^{1}$ \\ and U Stroth ${ }^{1,2}$ \\ ${ }^{1}$ Institut für Plasmaforschung, Universität Stuttgart, Pfaffenwaldring 31, 70569 \\ Stuttgart, Germany \\ ${ }^{2}$ Max-Planck-Institut für Plasmaphysik, EURATOM-Assoziation, Boltzmannstr. 2, \\ 85748 Garching, Germany \\ E-mail: Gregor.Birkenmeier@ipp.mpg.de
}

\begin{abstract}
The three-dimensional structure of drift-wave turbulence and turbulent transport is investigated in plasmas of the stellarator experiment TJ-K. By means of two poloidal Langmuir probe arrays placed at different toroidal positions, density and potential fluctuations are recorded simultaneously at 128 positions on a single flux surface. From this data, the spatial drift-wave turbulence pattern including perpendicular and parallel structure sizes are obtained using a cross-correlation technique. A comparison with the magnetic field structure indicates an initially perfect alignment of turbulent structures with magnetic field lines. Passing over regions with different field line pitches according to the local variation of the rotational transform, however, results in a measured displacement of turbulent structures with respect to the field lines during their radial propagation. A reduction of the perpendicular correlation lengths in regions of high absolute values of local magnetic shear is found. Prominent and poloidally narrow turbulent transport maxima are measured at different toroidal positions. They are connected by the magnetic field lines and located in regions of negative normal curvature. The poloidal propagation pattern of turbulent structures and the exact position of the transport maximum depend on the magnetic field direction.
\end{abstract}

PACS numbers: 52.25.Xz, 52.25.Fi, 52.35.Ra, 52.55.Hc

Submitted to: Plasma Phys. Control. Fusion

$\ddagger$ Present address: Max-Planck-Institut für Plasmaphysik, EURATOM-Assoziation, Boltzmannstr. 2, 85748 Garching, Germany 


\section{INTRODUCTION}

Drift-wave turbulence $[1,2]$ is known to contribute significantly to the loss of energy and particles at the edge of magnetically confined plasmas. Therefore, the progress in the magnetic confinement of plasmas is inevitably related to our understanding of driftwave turbulence. In addition to turbulence driving or reducing background parameters like pressure gradients and radial electric fields [3], the magnetic field geometry is able to modify the spatio-temporal appearance of drift-wave turbulence [4]. Local parameters of the magnetic field can generate an additional drive of instability and lead to spatial inhomogeneities of turbulent transport. Hence, the modification of turbulence by choosing an appropriate magnetic field geometry opens a promising route for future confinement optimization.

Early models describe drift-wave turbulence in simple geometry with constant magnetic field [5]. More realistic models, however, have to take into account the magnetic field geometry of magnetic confinement experiments. Especially in complex stellarator configurations, the local properties of the magnetic field, like curvatures and field-line shear, have to be included into the models in order to describe the structure and dynamics of turbulence appropriately. Different approaches like a ballooning-mode formalism $[6,7,8,9,10,11]$, a full three-dimensional analysis of unstable modes on a flux surface $[12,13,14,15]$ and non-linear fluid $[16,17,18]$ or gyrokinetic $[19,4,20,21]$ simulations point to significant effects of the local magnetic field geometry on the spatial structure and the stability of drift modes. Theoretical studies indicate that turbulent transport can be reduced by more than a factor of 2 if an appropriate shaping procedure considering local magnetic field properties is applied [22].

In parallel to these promising theoretical developments, only a few experimental studies on the spatial properties of turbulence depending on local magnetic parameters exist. Measured poloidal asymmetries in the fluctuation amplitudes [23, 24, 25, 26, $27,28,29,30,31]$ point to effects of field-line curvature at the outboard side of the flux surface in tokamaks. Recent experiments in a stellarator configuration confirm the poloidal asymmetries in fluctuations and turbulent transport $[32,33]$ in the region of negative normal curvature ("unfavorable curvature"), but point to further local effects in connection with the geodesic curvature. With the aid of multi-Langmuir probe arrays [34], it was possible to resolve the perpendicular shape of turbulent structures in the edge or scrape-off layer of stellarator plasmas in detail [35, 36, 37, 38]. In addition, some works $[39,40,41]$ extended the three-dimensional picture of drift-wave turbulence by measurements of the parallel structure and relating it to the three-dimensional pathway of a magnetic field line. The studies presented in the paper at hand go beyond these works and reveal the three-dimensional structure of turbulence and turbulent transport on a flux surface simultaneously measured by means of two 64-multi-probe arrays. By cross-correlation analyses, the spatial appearance and dynamics of turbulent structures are investigated and related to local properties of the magnetic field geometry. Direct measurements of turbulent transport at 160 positions on a single flux surface are 
performed in order to identify most unstable regions. The following points will be addressed in detail:

- Turbulent structures evidently originate field-aligned in the steep density gradient region and propagate radially outward causing a poloidal displacement relative to the field line due to magnetic shear.

- Parallel correlation lengths of several meters are found and exhibit an asymmetry with respect to the corresponding reference probe.

- Poloidal correlation lengths only amount to $1.3 \%$ of the parallel lenghts and are reduced in regions of high absolute local magnetic shear.

- Measured poloidal velocities of turbulent structures are comparable to the electron diamagnetic drift but reverse sign at specific regions at the top and bottom of the flux surface. The location of these regions changes with magnetic field direction.

- The turbulent transport maxima measured on a flux surface in unprecedented detail are located in the bad curvature region and are connected along the field line. The maxima positions change with magnetic field direction pointing to an influence of the geodesic curvature on turbulent transport.

The paper is organised as follows: In Sec. 2, the stellarator experiment, its diagnostics and magnetic field structure is described. After introducing the cross-correlation technique in Sec.3, the three-dimensional appearance and dynamics of turbulent structures are presented in Sec.4. Based on the knowledge of the spatial structure of turbulence, the turbulent particle transport measurements are discussed in Sec. 5 followed by a summary in Sec. 6 .

\section{Experimental Setup}

All presented measurements are performed at the stellarator experiment TJ-K [42]. A schematic view of TJ-K is shown in Fig. 1 (top). The coil system corresponds to an $l=1, m=6$ torsatron and has a major plasma radius of $R_{0}=0.6 \mathrm{~m}$ and a minor plasma radius of about $a=0.1 \mathrm{~m}$. TJ-K is a low-temperature plasma experiment with cold ions [43], and with plasma parameters dimensionally similar to the edge of larger fusion experiments [44]. Due to the accessibility for Langmuir probes in the whole confinement region, it is an ideal test bed for turbulence investigations [34]. In comparison with fluid simulations [45], the indicators for electrostatic driftwave turbulence like a small density-potential cross-phase, a propagation of turbulent structures into electron-diamagnetic drift direction [42], a finite parallel wave length [41], and low magnetic field fluctuations [46] have been identified. Based on these results, the scaling of turbulent structures [36], the dynamics of blobs in the scrape-off layer [38], the modification of turbulent transport by $E \times B$-shear flows [37], and the dynamics and energy transfer of zonal flows have been investigated [47, 48, 49, 50, 51].

For the turbulence investigations presented in this paper, the two multi-Langmuir probe arrays shown in Fig. 1 (bottom) are used. Each array consists of 64 probes 

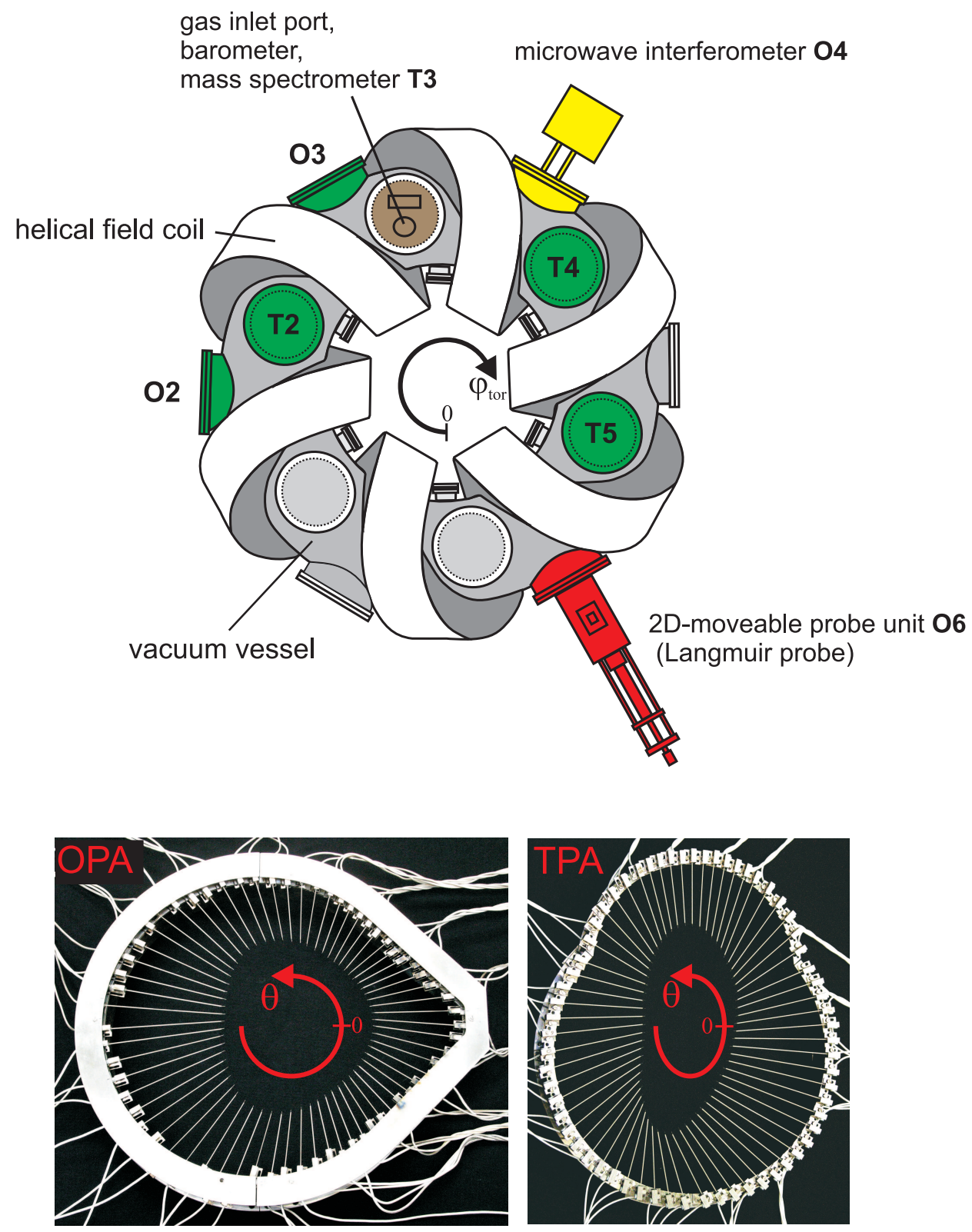

Figure 1. Schematic view on TJ-K. The Outer Port Array (OPA, photograph on the left) is installed at the outer port O2 and O3, the Top Port Array (TPA, photograph on the right) is successively installed at the top ports T5, T2, T4.

with $2 \pm 0.25 \mathrm{~mm}$ tip length and a poloidal distance between adjacent probe tips of $d x=8 \pm 0.5 \mathrm{~mm}$. All probe tips are aligned to a single flux surface located $1.5 \mathrm{~cm}$ inside the last closed flux surface (LCFS). The magnetic configuration is fixed by the current ratio of $R_{\mathrm{v} / \mathrm{h}}=57 \%$ (ratio between vertical and helical field-coil current). Since the so-called Outer Port Array (OPA) is installed at a symmetry plane at an outer port (see Fig. 1, left), and the Top Port Array (TPA) at a top port (see Fig. 1, right), the probe arrangements differ according to the local shape of the three-dimensional flux surface (cp. Fig. 2). While the poloidal cross-section of the flux surface at the outer 
ports has a triangular shape and is up-down symmetric, the flux surface at the top ports lacks in symmetry and is elliptically shaped.

Every 60 in toroidal direction, the magnetic field is topologically equivalent due to the sixfold toroidal symmetry of the coil system. This allows to successively install the probe arrays at different ports in order to measure poloidal profiles of turbulence properties at different toroidal positions. Therefore, the OPA was installed at the outer ports $\mathrm{O} 2$ and O3, and the TPA at the top ports T2, T4, and T5 (Fig. 1). If not otherwise stated, results from the OPA are acquired at port O2, and results from TPA at T5.

Since the magnetic field structure is able to modify the spatial turbulence pattern, some characteristics of the magnetic configuration in TJ-K are presented in the following. The shape of the flux surfaces of TJ-K is well known from flux surface measurements by a fluorescence rod technique and comparisons with a field line tracing code (like in Ref. [52]). From these measurements, a realistic coil model was developed in order to take into account error fields induced by a displacement of the vertical field coils by a few millimeters. For comparison with the measurements, the magnetic properties on a TJ-K flux surface corresponding to a current ratio of $R_{\mathrm{v} / \mathrm{h}}=57 \%$ are calculated with a field line tracing code. Figure 2, a), shows the magnetic field strength $B$ color coded on a whole flux surface depending on the toroidal angle $\varphi_{\text {tor }}$ and poloidal angle $\theta$ of a right-handed toroidal coordinate system. A three-dimensional visualization of the flux surface including the variation of $B$ is depicted in Fig. 2, b). The sixfold toroidal symmetry due to the course of the helical coil can be seen in the regions of high $B$ (red shaded). Hence, the high-field side is not always at the inboard side, but e.g. for the top ports on the bottom. Due to this magnetic field structure, collisionless particles trapped between maxima of the magnetic field strength $B$ [53] can exist (black and white arrows).

A second local magnetic field quantity which is related to the curvature drive of the drift-wave instability is the normal curvature $\kappa_{n}$ shown in Figs. 2, c) and d):

$$
\kappa_{n}=\boldsymbol{\kappa} \cdot \frac{\boldsymbol{\nabla} \psi}{|\nabla \psi|}
$$

with the field line curvature vector $\boldsymbol{\kappa}$ and the toroidal magnetic flux $\psi$. Usually, a negative normal curvature is called "unfavorable" since it appears in growth rates of interchange modes [54] and micro instabilities [8]. As can be seen from Figs. 2, c) and d), the maximum unfavorable curvature is mainly found at the outboard side (white), and oscillates toroidally according to the sixfold toroidal symmetry. In contrast to a tokamak geometry where unfavorable curvature is only found at the outboard side, in TJ-K, the negative normal curvature region at top (bottom) ports is found at the top (bottom) of the flux surface.

Since the considered configuration at current ratio $R_{\mathrm{v} / \mathrm{h}}=57 \%$ is almost shearless, the local magnetic shear [11]

$$
S:=\frac{(\mathbf{B} \times \nabla \psi) \cdot \boldsymbol{\nabla} \times(\mathbf{B} \times \boldsymbol{\nabla} \psi)}{2 \pi|\nabla \psi|^{4}}
$$




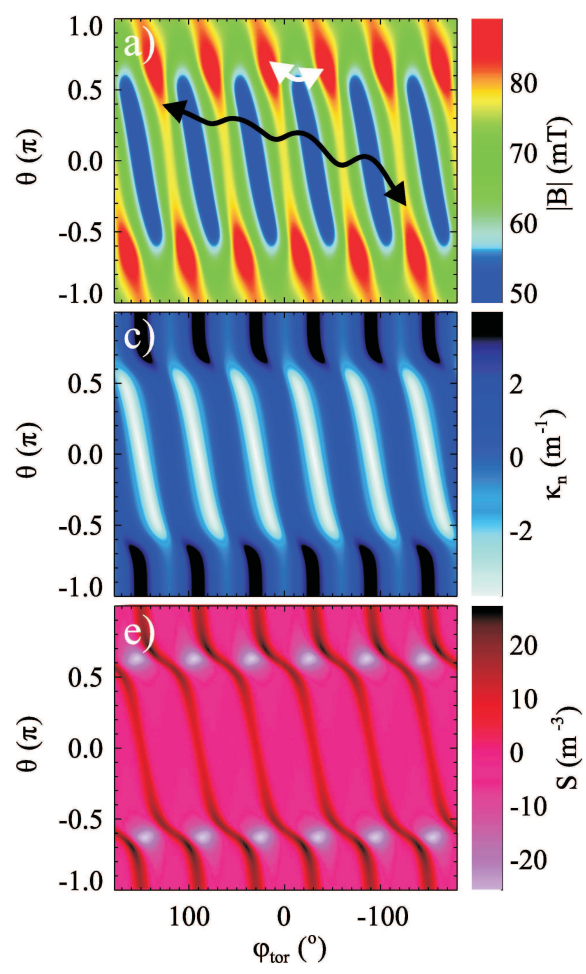

b)

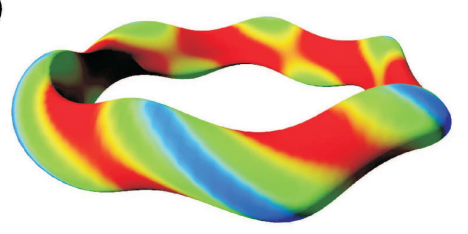

d)

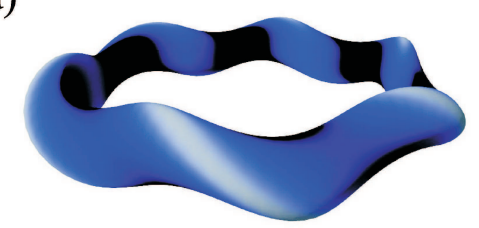

f)

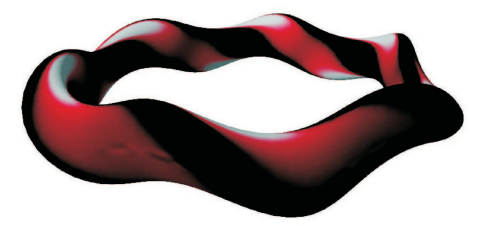

Figure 2. Geometry of a TJ-K flux surface in the $\varphi-\theta$-plane (left) and in a 3Dvisualization (right). The sixfold toroidal symmetry is visible in the magnetic field strength $B(\mathrm{a}, \mathrm{b})$, the normal curvature $\kappa_{n}(\mathrm{c}, \mathrm{d})$ and the local magnetic shear $S(\mathrm{e}, \mathrm{f})$. Due to its geometry, helically (white line in a) and toroidally trapped particles (black line in a) could exist.

( $2 \pi S$ agrees with the local magnetic shear introduced in Ref. [54]) dominates over the global magnetic shear $s=\langle S\rangle=-0.0327 \mathrm{~m}^{-3}(\langle\cdot\rangle$ is a flux surface average). Thus, the local magnetic shear rather than global average shear is expected to localize unstable drift modes $[7,8]$ in regions of low shear. Figures 2, e) and f), show the local magnetic shear on the TJ-K flux surface. At the top and the bottom of the flux surface around $\theta \approx \pm 0.6 \pi$, the local magnetic shear reaches its highest absolute values up to $|S| \approx 27$ $\mathrm{m}^{-3}$. Thus, TJ-K possesses an "shear edge" at the top and bottom of the flux surface.

In helium discharges with a neutral gas pressure of $p_{0}=15 \mathrm{mPa}$ heated at the upper-hybrid resonance [55] with $2 \mathrm{~kW}$ from a $2.45 \mathrm{GHz}$ microwave system, densities of $n \approx 1.0 \cdot 10^{17} \mathrm{~m}^{-3}$ and electron temperatures of $T_{e} \approx 9 \mathrm{eV}$ are achieved at the probe tip positions of the arrays corresponding to $r=6.5 \mathrm{~cm}$ (vertical dashed line) as shown for discharge \#6750 in Fig. 3. While the density profile (Fig. 3, a) increases towards the magnetic axis at $r=0 \mathrm{~cm}$, the electron temperature profile (Fig. 3, b) is flat in the confinement region. The ion temperature profile is flat as well but reaches much lower values around $T_{i} \approx 0.1 \mathrm{eV}[43]$. Since the equilibrium profiles are the same in discharges 
with and without the probe arrays installed, it is assumed that their perturbation on the plasma dynamics is small which is also supported by turbulence studies performed with a high speed camera in cases with and without the presence of Langmuir probes. For the considered discharges, the drift scale $\rho_{s}=\sqrt{m_{i} T_{e}} /(e B)$, which is relevant for turbulent structure sizes, is in the centimetre range and, therefore, exceeds by far the ion Larmor radius $\rho_{i}=\sqrt{m_{i} T_{i}} /(e B)$ and electron Larmor radius $\rho_{e}=\sqrt{m_{e} T_{e}} /(e B)$ as shown in Fig. 3, c). Ion-saturation current and floating potential signals, $I_{\mathrm{i}, \mathrm{sat}}$ and $\phi_{\mathrm{fl}}$, respectively, are recorded with the two poloidal Langmuir probe arrays for $1 \mathrm{~s}$ with a time resolution of $1 \mathrm{MHz}$. For the turbulence investigations only the AC-part of the signals is used. As it was shown in previous studies, the floating-potential fluctuations are proportional to the plasma-potential fluctuations $\tilde{\phi}_{\mathrm{fl}} \propto \tilde{\phi}_{\mathrm{p}}$, and the ion-saturation current fluctuations are proportional to density fluctuations $\tilde{I}_{\mathrm{i} \text {,sat }} \propto \tilde{n}$ due to a flat temperature profile and, hence, negligible temperature fluctuations [56].
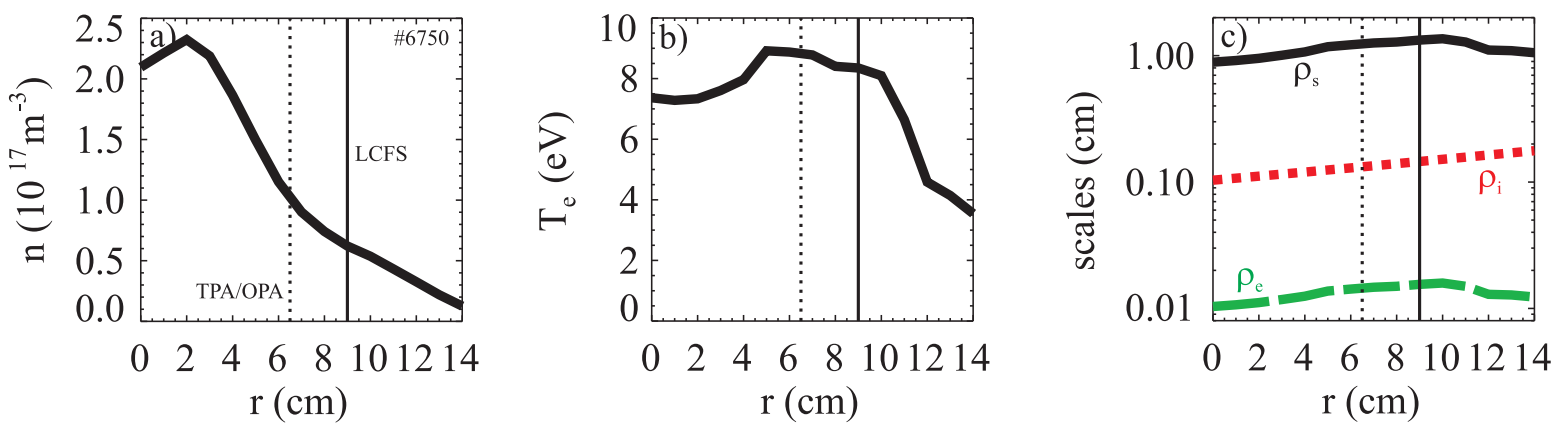

Figure 3. Radial profiles of density (a), electron temperature (b), and perpendicular scales (c) namely the drift scale $\rho_{s}$ (solid), ion Larmor radius $\rho_{i}$ (dotted), and electron Larmor radius $\rho_{e}$ (dashed). The vertical solid (dashed) line indicates the radial location of the LCFS (probe arrays).

\section{3D STRUCTURE OF DRIFT-WAVE TURBULENCE}

In order to reveal the spatial shape and the dynamics of coherent structures within the turbulent fluctuations, a cross-correlation analysis is performed. In this way, the characteristic and statistically significant properties of turbulent structures are accessible. Therefor, a first signal $x\left(t_{i}\right)$ from a reference probe and an arbitrarily selected second probe signal $y\left(t_{i}\right)$ are chosen $\left(i \in[1, N]\right.$ with $\left.N=1024000, t_{i} \in[0,1.024] \mathrm{s}\right)$, and the cross-correlation function

$$
\mathrm{CC}_{x y}(\Delta t)=\sum_{i=1}^{N} \frac{x\left(t_{i}\right) y\left(t_{i}+\Delta t\right)}{\sigma_{x} \sigma_{y}}
$$

depending on the time lag $\Delta t$ between the signals is calculated. $\sigma$ is the standard deviation of the signal. Hence, information on common features in the two signals is obtained: The amplitude $\mathrm{CC}_{x y} \in[-1,1]$ is maximum for a high correlation, negative for an anti-correlation, and zero for no correlation (e.g. for two white-noise signals). A 
reasonable high correlation maximum at a finite and positive time lag $\Delta t=t_{m}$ indicates a signal content in $x(t)$ appearing similarly in $y(t)$ at a time $t_{m}$ later.

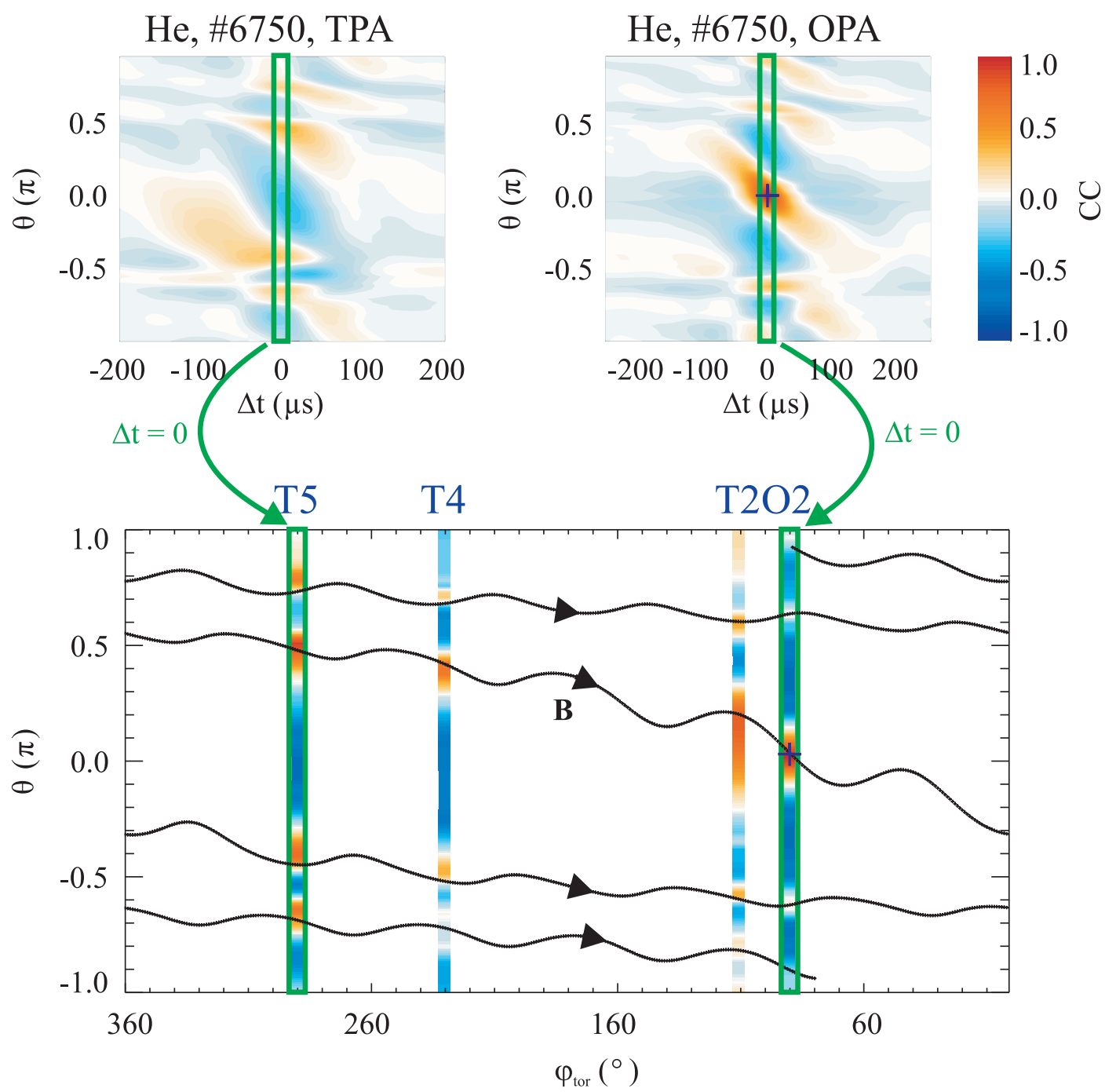

Figure 4. Cross-correlation functions $\mathrm{CC}_{x y}(\Delta t, \theta)$ of ion-saturation current signals depending on probe position $\theta$ with reference probe at the outboard side of OPA (cross) reveal the spatio-temporal properties of coherent structures (top). Correlation data taken at time $\Delta t=0$ from the TPA (top, left) and the OPA (top, right) is put into the $\varphi-\theta$-plane of the considered flux surface (bottom). The correlation maxima are connected via the magnetic field line (black).

Figure 4, top, shows cross-correlation functions calculated from the ion-saturation current signals $\widetilde{I}_{\mathrm{i} \text {,sat }}(t)$ from the TPA (left) and the OPA (right). A reference signal $x(t)=\widetilde{I}_{\mathrm{i}, \mathrm{sat}}^{\text {ref }}(t)$ at the outboard side of the OPA is chosen (cross in Fig. 4) and correlated with all probes of the OPA and all probes of the TPA. The results are contour maps $\mathrm{CC}_{x y}(\Delta t, \theta)$ depending on the time lag $\Delta t$ according to Eq. 3, and, in addition, on the poloidal probe position of the considered probe signal $y(t)=\widetilde{I}_{\mathrm{i} \text {,sat }}(t)$ expressed in $\theta \in[-\pi, \pi] . \theta$ is a poloidal angle counted counter-clock wise starting at the inboard side $(\theta=0$ corresponds to the outboard side in the equatorial plane, $\theta=\pi / 2$ to the top and 
$\theta=-\pi / 2$ to the bottom of the flux surface).

The spatio-temporal pattern of the coherent structures in Fig. 4, top, exhibits diagonal ribbon-like regions with increased correlation levels corresponding to a poloidal propagation of poloidally elongated structures (cp. Sec. 4), and several local maxima. The maximum value of $\mathrm{CC}_{x y}=1$ is achieved at the outboard side of OPA $(\theta=0)$, where $y(t)=x(t)$ (the auto-correlation of the reference probe signal), and minimum values are about $\mathrm{CC}_{x y} \approx-0.3$. Values of more than $\mathrm{CC}_{x y} \approx 0.4$ are also achieved at the TPA, although it is about $2 \mathrm{~m}$ apart from the reference probe indicating a parallel connection between toroidally separated regions. Obviously, the turbulent structures have a poloidal mode number of $m=4$ resulting in four local maxima at each array position. This is possibly related to the rotational transform of $t=0.227 \approx \frac{1}{4}$ and a finite parallel wave number [41] preferring a quasi-coherent $m=4$ mode. Furthermore, the life time of turbulent structures defined as the decay time of the auto-correlation function down to $\mathrm{CC}_{x x}=0.5$ is of the order of $\tau \approx 25 \mu \mathrm{s}$.

In order to further describe the spatial structure of the quasi-coherent modes, we focus on the cross-correlation functions at time lag $\Delta t=0$ in the following. Figure 4 , bottom, shows the cross-correlation function $\mathrm{CC}_{x y}(0, \theta)$ of ion-saturation current signals from the OPA installed at port O2 (corresponding to a toroidal angle of $\varphi_{\text {tor }}=90$ ) and the TPA installed consecutively at different top ports T2 $\left(\varphi_{\text {tor }}=110\right)$, T4 $\left(\varphi_{\text {tor }}=230\right)$ and T5 $\left(\varphi_{\text {tor }}=290\right)$ represented in a $\varphi-\theta$-plane covering the flux surface. The measurements are performed in separate discharges due to the relocation procedure of the TPA retaining the reference probe position at the outboard side of the OPA (cross in Fig. 4) at identical discharge parameters. Despite the fact that the data is taken from different discharges, the poloidal correlation $\mathrm{CC}_{x y}(0, \theta)$ at different ports features the $m=4$ mode structure. For comparison, the location of a magnetic field line in the $\varphi-\theta$-plane is calculated with a field line tracing code (black line in Fig. 4 , bottom). It is obvious that the local maxima of the poloidal correlation fit almost perfectly with the positions of the field line. This unambiguously illustrates the parallel interconnection of toroidally separated regions, and indicates an elongation of turbulent structures over $15 \mathrm{~m}$ parallel to the field line or more than four toroidal turns.

Because of the matching of the correlation function maxima with the magnetic field line, it is expedient to interpolate the correlation functions in parallel direction in order to obtain a full correlation map in the $\varphi$ - $\theta$-plane. Therefore, the values of the correlation functions are linearly interpolated along the magnetic field line shown in Fig. 4 continued to about 170 toroidal turns. This corresponds to a total segment length of $l_{\text {tot }}=650 \mathrm{~m}$ where $l$ is a field line coordinate in $\mathrm{m}$ starting at the reference probe position. Due to a non-rational rotational transform $t$, this field line segment covers the whole flux surface with a sufficient spatial resolution. The result of this interpolation is shown in Fig. 5. The parallel mode structure along $l$ around the correlation maximum as shown for a segment in Fig. 5, a) has a sharply declining peak with a socket followed by relatively low and oscillating correlation values below $30 \%$. The parallel length at $50 \%$ correlation level is $l_{\|, 50 \%}=2.9 \mathrm{~m}$ while the width of the socket (distance between 

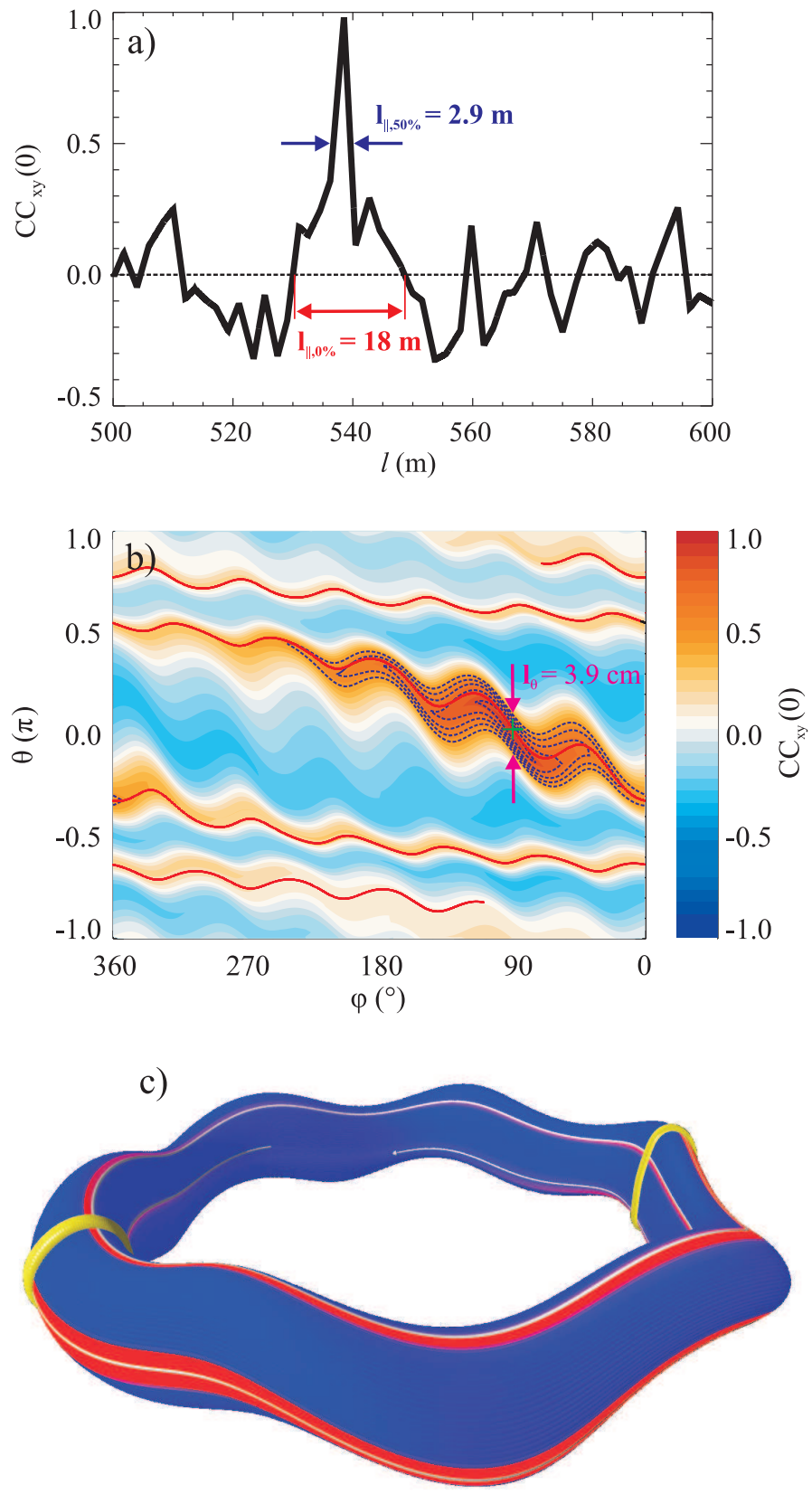

Figure 5. Linear interpolation of the cross-correlation function $\mathrm{CC}_{x y}(0)$ along the magnetic field line coordinate $l$ resolves the parallel structure of a quasi-coherent mode (a). This allows to reconstruct the full spatial structure of a quasi-coherent mode on a flux surface (b). In a 3D-view (c), the coherent drift-modes (red) measured with the probe arrays (yellow) appear aligned along the field lines (white). 
the zero-crossings) reaches more than the sixfold length $\left(l_{\|, 0 \%}=18 \mathrm{~m}\right)$. This is larger than the spatial resolution in parallel direction given by the averaged connection length between parallel connected probes of $\bar{L}_{\text {con }}=1.98 \mathrm{~m}$. Similar values have already been measured in former studies by means of a different analysis [41], and confirms the result of finite parallel wave numbers in agreement with the drift-wave scheme [2].

It should be noted that there is an asymmetry in the parallel correlation: the distance from the correlation maximum to the $50 \%$ level into magnetic field direction is only $l_{\|, \text {co }}=1.2 \mathrm{~m}$ while the corresponding distance against the magnetic field direction is $l_{\|, \text {ctr }}=1.7 \mathrm{~m}$, i.e., only $40 \%$ of the parallel correlation length is extended into magnetic field direction. In other words, there is a lower correlation into magnetic field direction than against it, although the connection length $L_{c o n}$ between the probes in co-direction $\left(L_{c o n}=1.88 \mathrm{~m}\right)$ is smaller than in counter-direction $\left(L_{c o n}=2.39 \mathrm{~m}\right)$ for the considered probe pairs. Thus, the correlation between parallel connected probes does not necessarily decrease with the connection length. Defining an asymmetry measure $\alpha_{\text {asym }}=\frac{l_{\|, \mathrm{co}}-l_{\|, \mathrm{cctr}}}{l_{\|}}$the asymmetry is quantified with $\alpha_{\text {asym }}=-0.17$. Negative values of $\alpha_{\text {asym }}$ correspond to an extension of the structure against the magnetic field direction. Even though $\left(l_{\|, \text {co }}-l_{\|, \text {ctr }}\right)$ is smaller than the resolution of the measurement, the sign of $\alpha_{\text {asym }}$ reliably indicates whether the correlation is higher in co- or counter-direction with respect to the reference probe.

A two-dimensional representation of the interpolated data reveals a full picture of a turbulent structure in the $\varphi-\theta$-plane (Fig. 5, b) on a flux surface. The highest correlation levels are achieved along the magnetic field line (red). More than $50 \%$ correlation (dashed contours) are achieved around the reference probe (green cross) at the outboard side of the OPA at $\theta=0$. A three-dimensional visualization of the correlation data in Fig. 5, c) nicely shows the alignment of the turbulent structure (red) to the magnetic field line (white). The structure passes the probe arrays (yellow) several times.

Around the reference probe position, also poloidal correlation lengths are determined. Choosing again a correlation level of $50 \%$, a poloidal correlation length of $l_{\theta, 50 \%}=3.9 \mathrm{~cm}$ is found. This highlights the anisotropy of the system since the ratio between poloidal and parallel correlation length is only $\frac{l_{\theta, 50 \%}}{l_{\|, 50 \%}}=1.3 \%$.

It should be emphasized that all so far presented results are obtained by choosing a single reference probe at the outboard side $(\theta=0)$ of the OPA. The same analysis with different reference probes chosen at the OPA provides additional values of parallel and poloidal correlation lengths, $l_{\|}$and $l_{\theta}$, depending on the reference probe position $\theta$. This poloidal dependence is examined in the following.

\subsection{Parallel correlation lengths}

For different reference-probe positions $\theta$, the parallel correlation length varies between $l_{\|, 50 \%} \approx 2.4 \mathrm{~m}$ at the top and the bottom of the flux surface, and $l_{\|, 50 \%} \approx 3.3 \mathrm{~m}$ at the inboard and outboard side (Fig. 6, top). The avarage value is $\bar{l}_{\|, 50 \%}=2.74 \pm 0.36$ 
m. The same analysis for hydrogen discharges yields a similar parallel length with $\bar{l}_{\|, 50 \%}=2.71 \pm 0.47 \mathrm{~m}$. This confirms former studies at TJ-K [41], which found the same parallel lengths for helium and hydrogen using a different method.

The parallel asymmetry of quasi-coherent drift-wave structures with respect to the correlation maximum depends on the reference probe position $\theta$, too. At the outboard side between $\theta=-0.5 \pi$ and $\theta=0.5 \pi$, the asymmetry factor $\alpha_{\text {asym }}$ is negative at forward field, as can be seen in Fig. 6, bottom (black circles). Hence, the structures at the outboard side are extended against the magnetic field direction. At the inboard side, this behaviour changes $\left(\alpha_{\text {asym }} \geq 0\right)$, and the main part of the structures is shifted into magnetic field direction. Since parallel correlation lengths are connected to parallel wave vectors $k_{\|}=2 \pi / \lambda_{\|}$, a preferred and poloidally dependent net propagation can be expected from this asymmetry.

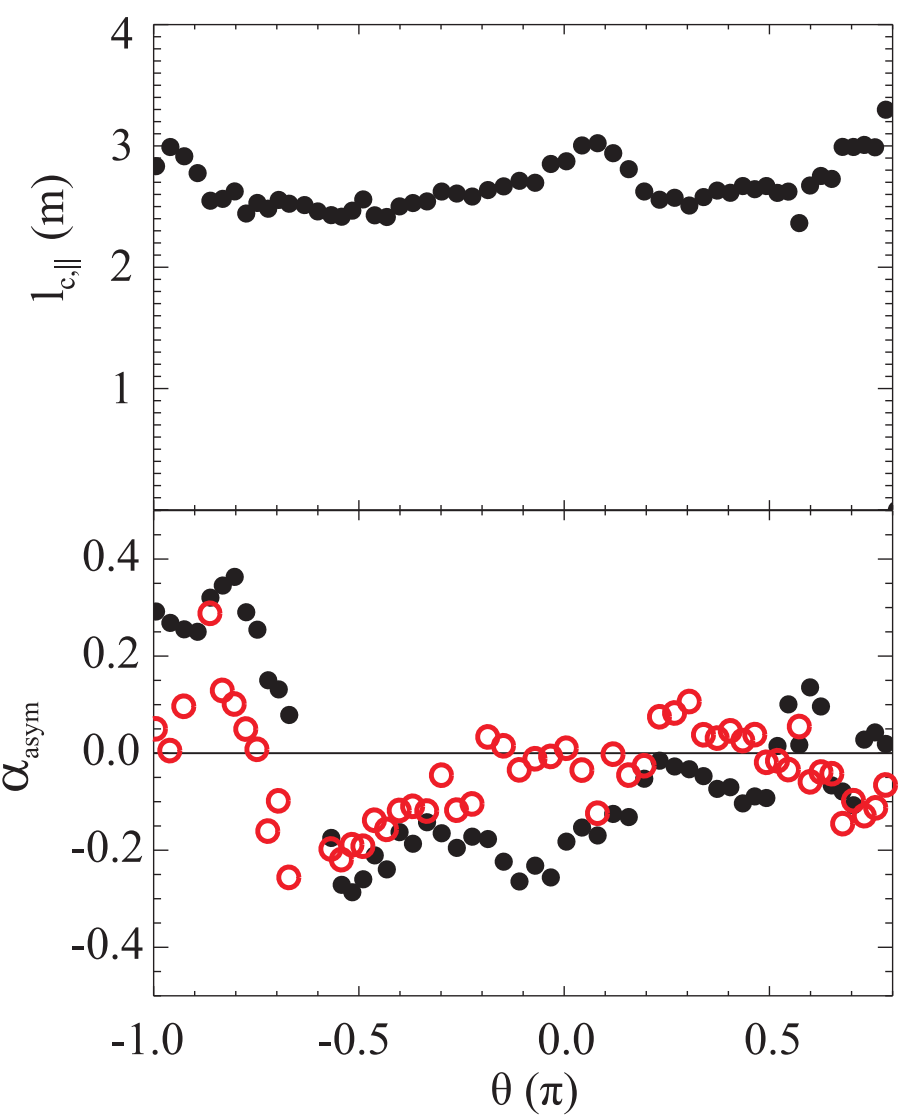

Figure 6. Parallel correlation lengths of turbulent density structures in the helium discharge \#6750 for different poloidal positions of the reference probe $\theta$ (top). The parallel elongation with respect to the correlation maximum is asymmetric and characterized by the asymmetry factor $\alpha_{\text {asym }}$ (bottom) at forward (black circles) and reversed field (open symbols).

At reversed field, the asymmetry factor (open symbols) shows a dependence similar to the forward field case. Due to the definition of $\alpha_{\text {asym }}$ with respect to the magnetic field this means, however, that the turbulent structures change their parallel extension in the lab frame. Thus, the asymmetry of parallel correlation lengths depends on the 
magnetic field direction. In both field directions, the asymmetry is lowest $\left(\alpha_{\text {asym }} \approx 0\right)$ around $\theta=-0.7 \pi$ and $\theta=0.5 \pi$ where the local magnetic shear $S$ is maximum (see Fig. 2) possibly "cutting" the turbulent structure in two parts of equal length.

\subsection{Poloidal correlation lengths}

Similarly to the parallel length, the poloidal correlation length $l_{\theta, 50 \%}$ shown in Fig. 7 (black circles) features a dependence of the reference probe position $\theta$, too. The poloidal correlation lengths at the OPA (left) exhibit a plateau at the outboard side with $l_{\theta, 50 \%} \approx 4.3 \mathrm{~cm}$. At the TPA (right), the correlation lengths are a bit larger with a maximum of $l_{\theta, 50 \%} \approx 5.2 \mathrm{~cm}$.

At both probe arrays, a poloidal dependence of the poloidal structure size is observable. It is maximum at the outboard side around $\theta=0$ and drops at the inboard side $(\theta= \pm \pi)$. In addition, prominent minima with values of about $l_{\theta, 50 \%} \approx 2 \mathrm{~cm}$ are found relatively localized around $\theta= \pm 0.6 \pi$ at the OPA and around $\theta \approx-0.6 \pi$, $\theta \approx 0.5 \pi$ and $\theta \approx 0.75 \pi$ at the TPA.
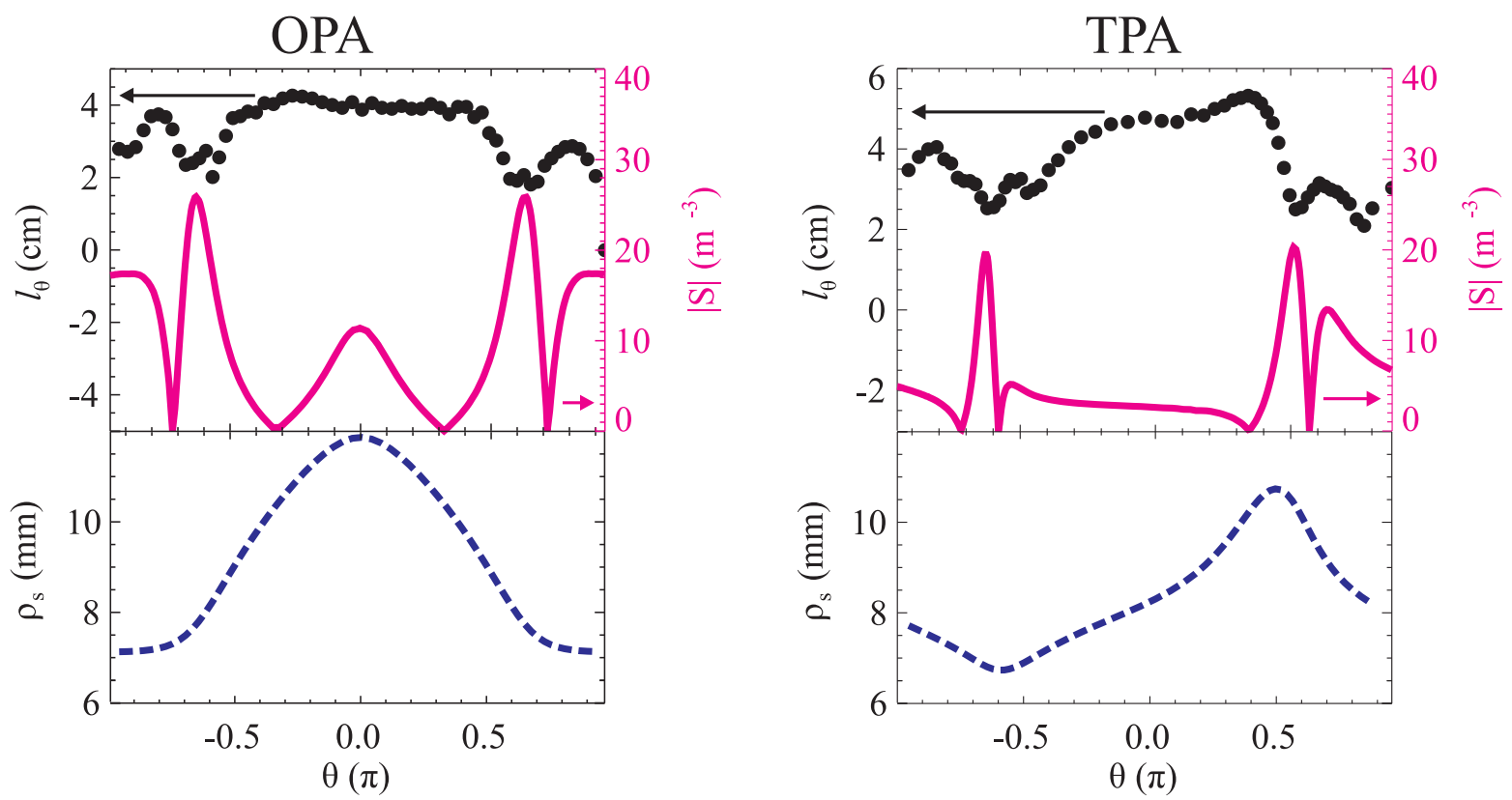

Figure 7. Poloidal correlation length $l_{\theta}$ (black circles) of turbulent density structures in a helium discharge for different poloidal positions $\theta$ of the reference probe at the OPA (left) and TPA (right). A very localized reduction of correlation lengths is found in regions of high absolute local magnetic shear $|S|$ (red solid line) while the drift scale $\rho_{s}$ (dashed line) possibly modifies the structure sizes on a larger scale.

What is the reason for the variation of the poloidal correlation length by a factor of two found at both array positions? Since the turbulent structures scale with the drift scale $\rho_{s}=\sqrt{m_{i} T_{e}} /(e B)$ with the ion mass $m_{i}$, the electron temperature $T_{e}$ and the magnetic field strength $B$ as it was shown in previous studies $[36,34]$, the poloidal dependence of $B$ could modify the perpendicular length scales. Indeed, $\rho_{s}$ has a 
maximum at the outboard side at the OPA and also a maximum at the top of the flux surface at the TPA as can be seen in Fig. 7, bottom (dashed line), and, therefore, roughly reflects the poloidal dependence of $l_{\theta}$. However, the plateau at the OPA, and the localized minima of $l_{\theta}$ at both arrays do not correspond to the poloidal dependence of $\rho_{s}$.

The local magnetic shear $S$ (see Eq. 2) is a candidate for reducing poloidal correlation lengths by tilting or decorrelating turbulent eddies [18]. Actually, in our measurements the reduced poloidal correlation lengths correlate with high values of absolute local magnetic shear as can be seen in Fig. 7, top (solid line). At the OPA, the highest absolute values of local magnetic shear are achieved at $\theta= \pm 0.63 \pi$, exactly where the lowest poloidal correlation lengths are found. Also at the TPA, local minima of poloidal correlation lengths are found at $\theta=-0.6 \pi$ and $\theta=0.5 \pi$ where the local magnetic shear has its local maxima.

The macrostructure of the perpendicular size of turbulent structures seems to be governed by the poloidal dependence of $\rho_{s}$. The localized reduction of $l_{\theta}$ at the top and bottom of the flux surface, however, is due to a local modification of structures by strong local magnetic shear $S$ irrespective of its sign.

\subsection{Structure displacement from field line}

The elongation of a turbulent structure along a field line is schematically indicated in Fig. 8, a). In general, the alignment is not perfect though. Thus, a turbulent structure detected at a particular reference probe at time lag $\Delta t=0$ is subject to a tilt with respect to the field line. In some toroidal distance, this tilt would be reflected in a displacement $\delta$ of the local correlation maximum relative to the crossing point of the field line from the reference probe.

From the probe data, the displacement $\delta$ is determined by the following procedure: A reference probe at the OPA (installed at port O2) is chosen. By a cross-correlation analysis the local maximum at the TPA (installed at port T4 and T5, respectively) is determined. The displacement $\delta$ is calculated as the difference between the local correlation maximum at the TPA and the poloidal position $\theta_{\text {TPA }}$, which is connected along a field line with the reference probe. Choosing different reference probes at the OPA, a variation of the displacement $\delta$ depending on the connection length $l_{\text {con }}$ (distance between a reference probe at the OPA and a parallel connected probe at $\theta_{\text {TPA }}$ at the TPA) is obtained (Fig. 8, b). Since the TPA consecutively is installed at port T4 and T5 with the OPA remaining fixed, different connection lengths $l_{\text {con }}$ between $1.35 \mathrm{~m}$ and $2.45 \mathrm{~m}$ are available. The measured displacement $\delta$ at T4 (\#7228, green circles) and T5 (\#6750, blue circles) shows a similar pattern for each toroidal measurement position starting with positive values of a few centimetres and decreasing to negative values. This is in contradiction to a simple picture like in Fig. 8, a), which implies a proportional increase of the displacement with connection length $\delta \propto l_{\text {con }}$ as can be derived from the drift-wave dispersion relation in slab geometry [41]. 

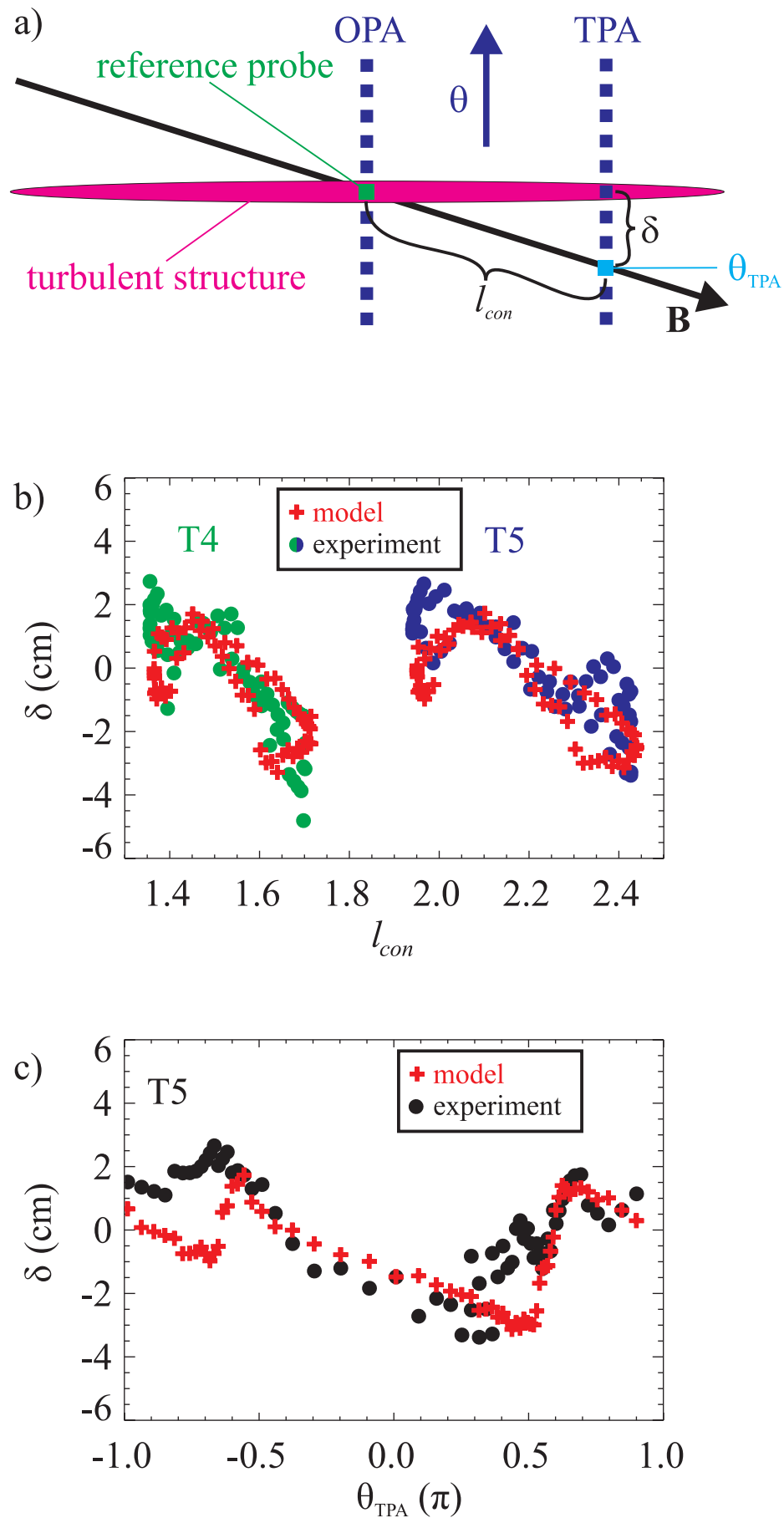

Figure 8. If a turbulent structure is not perfectly aligned to a magnetic field line, a displacement $\delta$ is measured at the TPA (a). The displacement $\delta$ measured at port $\mathrm{T} 4$ and T5 (circles) depends in a complex manner on the connection length $l_{\text {con }}(\mathrm{b})$ and on the poloidal angle $\theta_{\text {TPA }}$ (c) at the TPA which is in agreement with a model (crosses) according to Fig. 9. 
As it is exemplarily shown in Fig. 8, c), the displacement $\delta$ also depends on the poloidal angle $\theta_{\text {TPA }}$ at the TPA (port T5). The displacement is negative at the outboard side $\left(-0.5 \pi<\theta_{\mathrm{TPA}}<0.5 \pi\right)$, and positive at the inboard side. It reaches values of up to $\delta \approx-3.4 \mathrm{~cm}$ which is in the range of the poloidal structure size (cp. Sec. 3.2).

a) Creation of a structure at $\Delta \mathrm{t}<0$

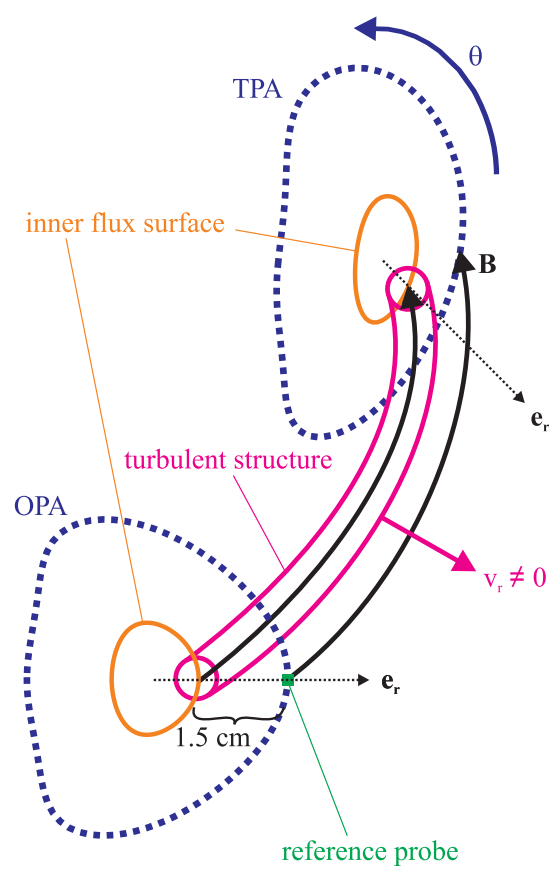

b) Detection of a structure at $\Delta \mathrm{t}=0$

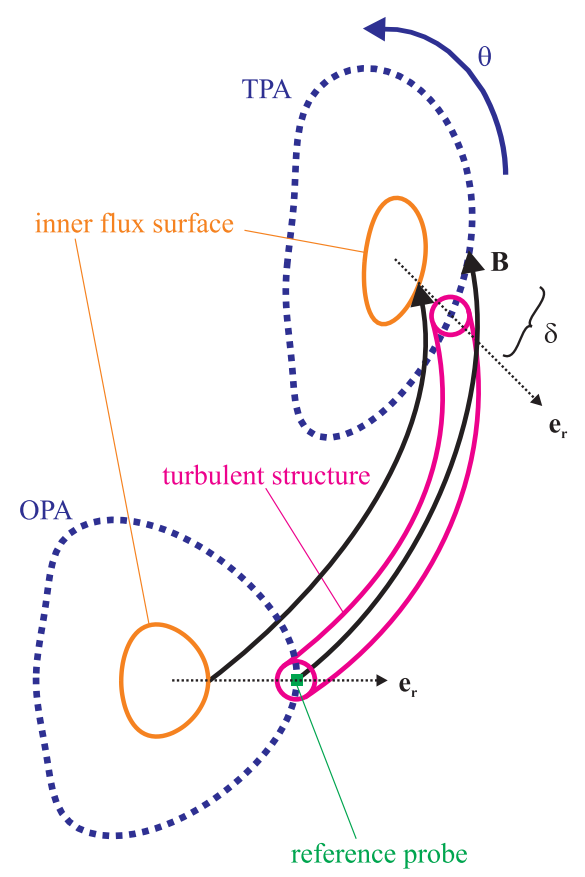

Figure 9. The measured displacement (cp. Fig. 8) is in agreement with a model, which assumes the origin of a parallel extended and perfectly field-aligned turbulent structure on a flux surface $1.5 \mathrm{~cm}$ inside the measurement flux surface at time $\Delta t<0$ (a). At time $\Delta t=0$, the turbulent structure propagates radially outward and touches the reference probe at the OPA (b). The displacement $\delta$ at the TPA is due to a different $t$ at the measurement flux surface.

Since the simple picture in Fig. 8, a), implying $\delta \propto l_{\text {con }}$ fails, another model in agreement with the data is established. The model starts from the premise that a turbulent structure originates from a flux surface $1.5 \mathrm{~cm}$ radially inside the measurement flux surface at a time $\Delta t<0$. Furthermore, it is assumed that the structure initially is perfectly aligned to the magnetic field line on the flux surface where it is created as it is shown in Fig. 9, a). Due to a radial outward propagation, the turbulent structure passes the reference probe at the OPA at a time $\Delta t=0$ (Fig. 9, b). At this time, however, the turbulent structure is not aligned to the field line anymore since the rotational transform $t$ at the measurement position differs from that at the initial flux surface resulting in a finite displacement $\delta$ between the turbulent structure and the field line on the measurement flux surface.

According to this model, the displacement $\delta$ can be calculated by a radial projection of the inner magnetic field line onto the $1.5 \mathrm{~cm}$ radially outward located measurement flux surface using a magnetic-field line code. The result is indicated by crosses in Fig. 8, 
b) and c). The shape of the calculated and measured pattern of $\delta$ depending on $l_{\text {con }}$ and $\theta_{\text {TPA }}$ as well as the absolute values are in good agreement. Furthermore, the validity of the model is supported by the fact that the maximum density gradient as a source of free energy for turbulence is found in a distance of $1.5 \mathrm{~cm}$ inside the measurement position.

In summary, the following explanation of the measured spatial structure of turbulence is found: In a region of maximum density gradient, the turbulent structures develop parallel extended and perfectly aligned to the magnetic field line. Subsequently, the turbulent structures propagate radially outward, and give rise to a displacement $\delta$ with respect to the field line due to different rotational transforms $t$ on the initial flux surface and the measurement flux surface. The structures are parallel extended by about $l_{\|} \approx 2.8 \mathrm{~m}$, and are asymmetric relative to the reference probe position into or against the magnetic field direction. The poloidal size of the turbulent structures $l_{\theta}$ of a few centimetres is roughly governed by the poloidal variation of the drift scale $\rho_{s}$ but strongly reduced in regions of high absolute magnetic shear $|S|$ possibly due to tilting.

\section{3D-DYNAMICS OF DRIFT-WAVE TURBULENCE}

Up to this point, only a static picture of drift-wave turbulence was investigated by means of the cross-correlation analysis. In the following, the time-dependent behavior is studied by variation of the time lag $\Delta t$ in the correlation function $\mathrm{CC}_{x y}(\Delta t, \theta)$.

Poloidal velocities are determined by cross-correlation of adjacent probes. For this purpose, the time lag $\Delta t=t_{m}$ at the maximum of the cross-correlation function is used to estimate the mean poloidal velocity of turbulent structures by

$$
v_{\theta}=\frac{d x}{t_{m}}
$$

with the probe distance $d x$. Variation of the reference probe position yields poloidal profiles of $v_{\theta}$ for both arrays, OPA and TPA, separately (see Fig. 10). Since it is known that TJ-K plasmas are dominated by drift-wave turbulence [35], it is expected [2] that turbulent structures propagate into the direction of the electron-diamagnetic drift

$$
v_{\mathrm{e}, \mathrm{dia}}=\frac{\nabla p \times \mathbf{B}}{\rho B^{2}}
$$

with pressure $p$, charge density $\rho$ and magnetic field B. Figure 10 shows the calculated electron-diamagnetic drifts (dashed line) and the measured poloidal velocities (solid line) from correlation studies at the array positions. In the present case, the background $E \times B$ flow is small compared to $v_{\mathrm{e}, \text { dia }}$.

At the outboard side, the measured velocities of about $v_{\theta} \approx 2 \mathrm{~km} / \mathrm{s}$ at forward field (Fig. 10, top) are within the statistical uncertainties (shaded region) in agreement with the electron diamagnetic drift for both arrays, and confirm results from earlier studies at TJ-K $[38,41]$. However, at the bottom of the flux surface $(-0.6 \pi<\theta<-0.4 \pi)$ and in a very narrow region around $\theta \approx 0.55 \pi$, the measured velocities significantly deviate from the expected values and even change sign. It means that the structures at the 

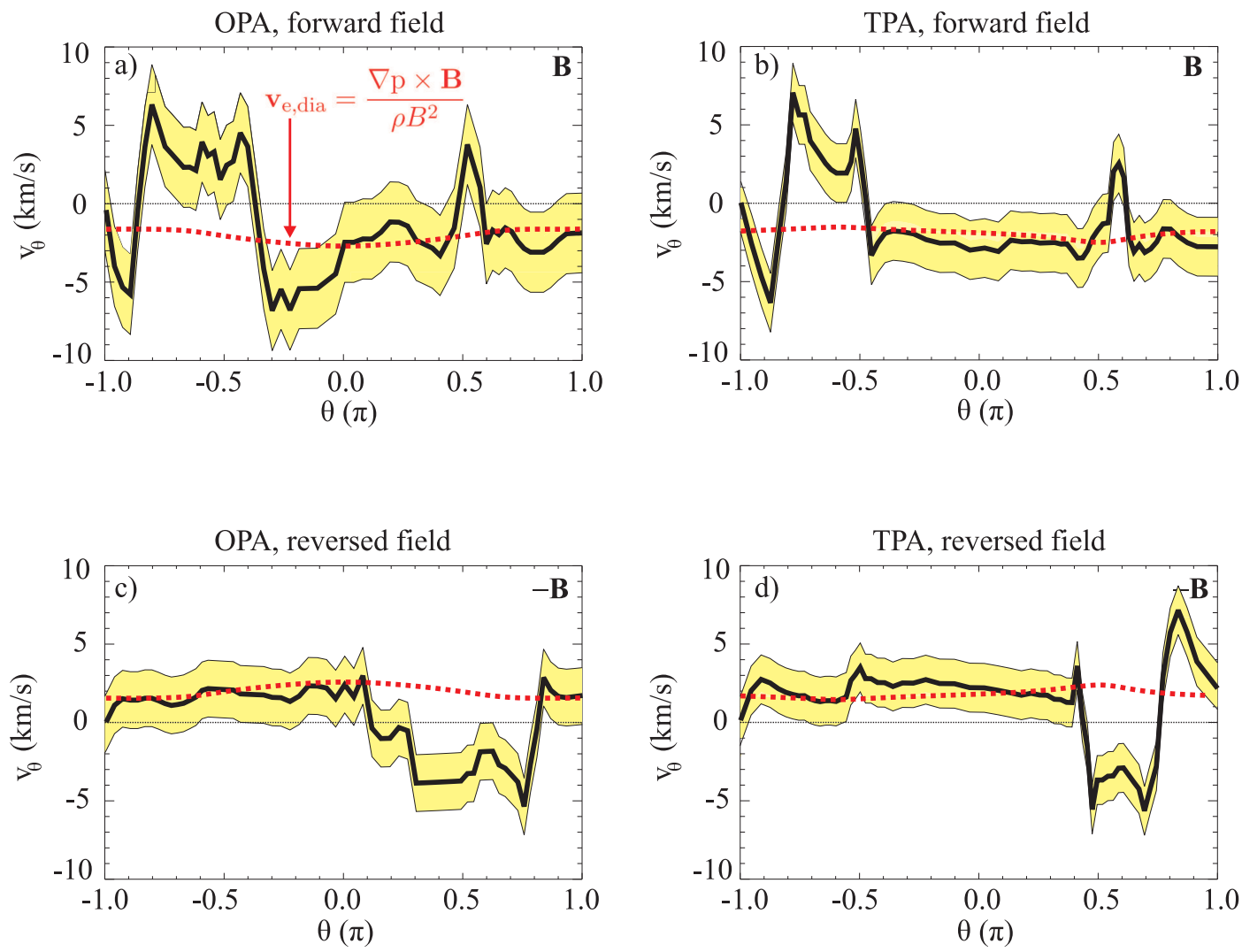

Figure 10. Measured poloidal velocity $v_{\theta}$ (solid line) depending on poloidal angle $\theta$ for the OPA (left) and the TPA (right) at forward (top) and reversed (bottom) magnetic field. The electron-diamagnetic drift is indicated in red (dashed).

bottom of the flux surface propagate into the opposite direction of the structures at the outboard side creating a complex propagation pattern.

Switching the magnetic field direction by reversing the current through the coils in TJ-K also reverses the drifts (Fig. 10, top). Again, there are regions where the measured velocities agree with the electron diamagnetic drift. But the sign reversal is now located at the top of the flux surface at $\theta \approx 0.5 \pi$. Again, a complex propagation pattern is found. It is partially in agreement with the drift-wave scheme at the outboard side but shows strong variations at the top of the flux surface. 


\section{Spatial Structure of Turbulent transport}

The correlation analysis in Sec. 3 revealed the spatial shape and the localisation of turbulent structures on a flux surface. However, the correlation analysis does not provide any information whether and where the coherent structures contribute to turbulent transport, which deteriorates the confinement. Therefore, dedicated transport studies were performed.

For this purpose, the local turbulent particle transport

$$
\Gamma=\left\langle\tilde{n} \tilde{v}_{r}\right\rangle_{t}=-\left\langle\tilde{n} \frac{\tilde{E}_{\theta}}{B}\right\rangle_{t} \propto\left\langle\tilde{I}_{\mathrm{i}, \mathrm{sat}} \frac{\left(\tilde{\phi}_{\mathrm{fl}, 1}-\tilde{\phi}_{\mathrm{fl}, 0}\right)}{2 B d x}\right\rangle_{t}
$$

as temporal average of density and radial velocity fluctuations, $\tilde{n}$ and $\tilde{v}_{r}=-\tilde{E}_{\theta} / B$, is estimated from measurements of fluctuations in ion-saturation current and floating potential. To this end, the probes on both the OPA and the TPA measure ion-saturation current and floating potential in alternating order. In this way, the poloidal electric field calculated by the finite difference $\tilde{E}_{\theta}=-\left(\tilde{\phi}_{\mathrm{fl}, 1}-\tilde{\phi}_{\mathrm{fl}, 0}\right) /(2 d x)$ is evaluated at the position of the ion-saturation current probe. The factor 2 takes into account twice the probe spacing $d x$ between the floating-potential probes $\tilde{\phi}_{\mathrm{fl}, 1}$ and $\tilde{\phi}_{\mathrm{fl}, 0}$. Negligible temperature fluctuations [56] justify the proportionalities $\tilde{\phi}_{\mathrm{fl}} \propto \tilde{\phi}_{\mathrm{p}}$, and $\tilde{I}_{\mathrm{i} \text {,sat }} \propto \tilde{n}$ in the last step of Eq. 6. The total error of the turbulent transport due to probe misalignment is estimated to be $13 \%$ [33].

The 128 probes of the OPA and the TPA allow to measure the turbulent transport simultaneously at 64 poloidal positions on a flux surface [33]. Putting the arrays consecutively at different toroidal positions at the ports $\mathrm{O} 2\left(\varphi_{\text {tor }}=90, \# 6750\right)$, O3 $\left(\varphi_{\text {tor }}=150, \# 7730\right), \mathrm{T} 2\left(\varphi_{\text {tor }}=110, \# 7192\right), \mathrm{T} 4\left(\varphi_{\text {tor }}=230, \# 7228\right)$, and $\mathrm{T} 5$ $\left(\varphi_{\text {tor }}=290, \# 6750\right)$, the turbulent transport is measured in a large area on 160 positions on the flux surface in total. The result of such a demanding procedure is shown in Fig. 11. Here, the transport is color coded (red corresponds to high transport levels) inset into the $\varphi$ - $\theta$-plane of the flux surface similar to Fig. 4 , bottom. As it was already shown elsewhere [33], the turbulent transport profile has one pronounced and narrow main maximum at every toroidal position. The maxima are mainly located at the outboard side between $-0.5 \pi<\theta<0.5 \pi$ in a region of negative normal curvature $\kappa_{n}$ ("unfavorable curvature") indicated in blue shading. In addition, the transport maxima are found in the vicinity of a magnetic field line, which passes the heating port B4 (star) at the bottom of the flux surface. 


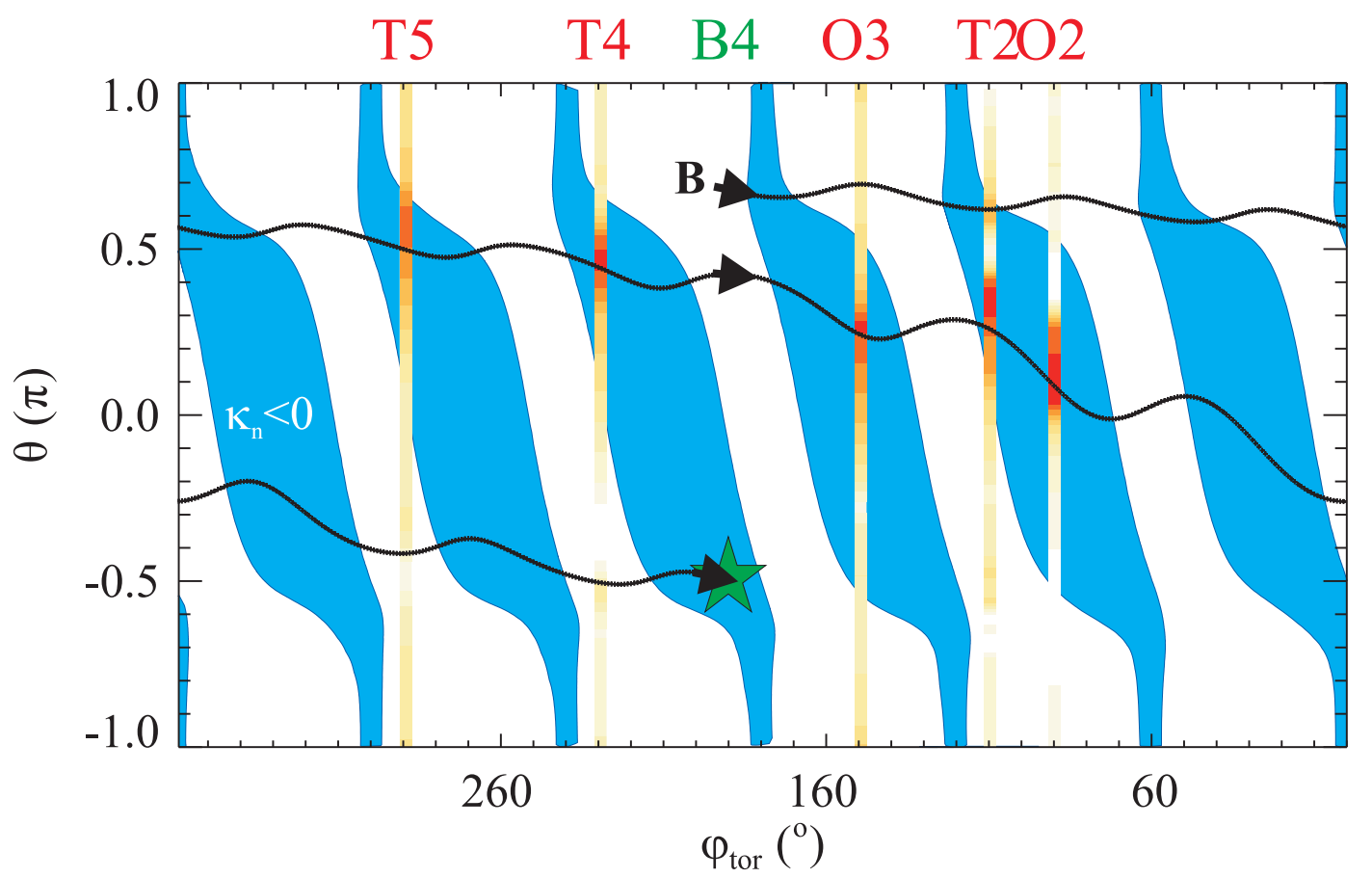

Figure 11. Poloidal turbulent transport profiles (red corresponds to high transport) measured at different toroidal positions on a flux surface at forward field. The transport maxima are located in a region of negative normal curvature $\kappa_{n}<0$ (shaded regions), and in the vicinity of a magnetic field line (solid) the heating position at port B4 (star).

The same measurement at reversed magnetic field results in a similar picture shown in Fig. 12: The pronounced and narrow turbulent transport maxima are located in an unfavorable curvature region at the outboard side, seemingly connected by the magnetic field line. But in the reversed field case, the transport maxima are poloidally shifted downward with respect to the forward case. Despite keeping the shape of the flux surface constant and choosing the same plasma parameters, the transport maxima are obviously influenced by the magnetic field direction.

Calculations of linear growth rates from a drift-wave model for TJ-K geometry [57] support the main instability drive of normal curvature $\kappa_{n}$ in agreement with the measurements. In addition, the theoretical growth rates are sensitive to the magnetic field direction due to the magnetic field dependence of the so-called geodesic curvature $[54,58,59]$

$$
\kappa_{g}=\boldsymbol{\kappa} \cdot\left(\frac{\boldsymbol{\nabla} \psi}{|\boldsymbol{\nabla} \psi|} \times \frac{\mathbf{B}}{B}\right) .
$$

Thus, the magnetic field direction dependence of the transport profiles indicates a contribution of this quantity to instability [33]. In particular, calculated local growth rates and measured transport profiles at the ports $\mathrm{O} 2$ and $\mathrm{T} 5$ showed a strong similarity [57].

Furthermore, the observed transport maxima in the vicinity of the field line point 


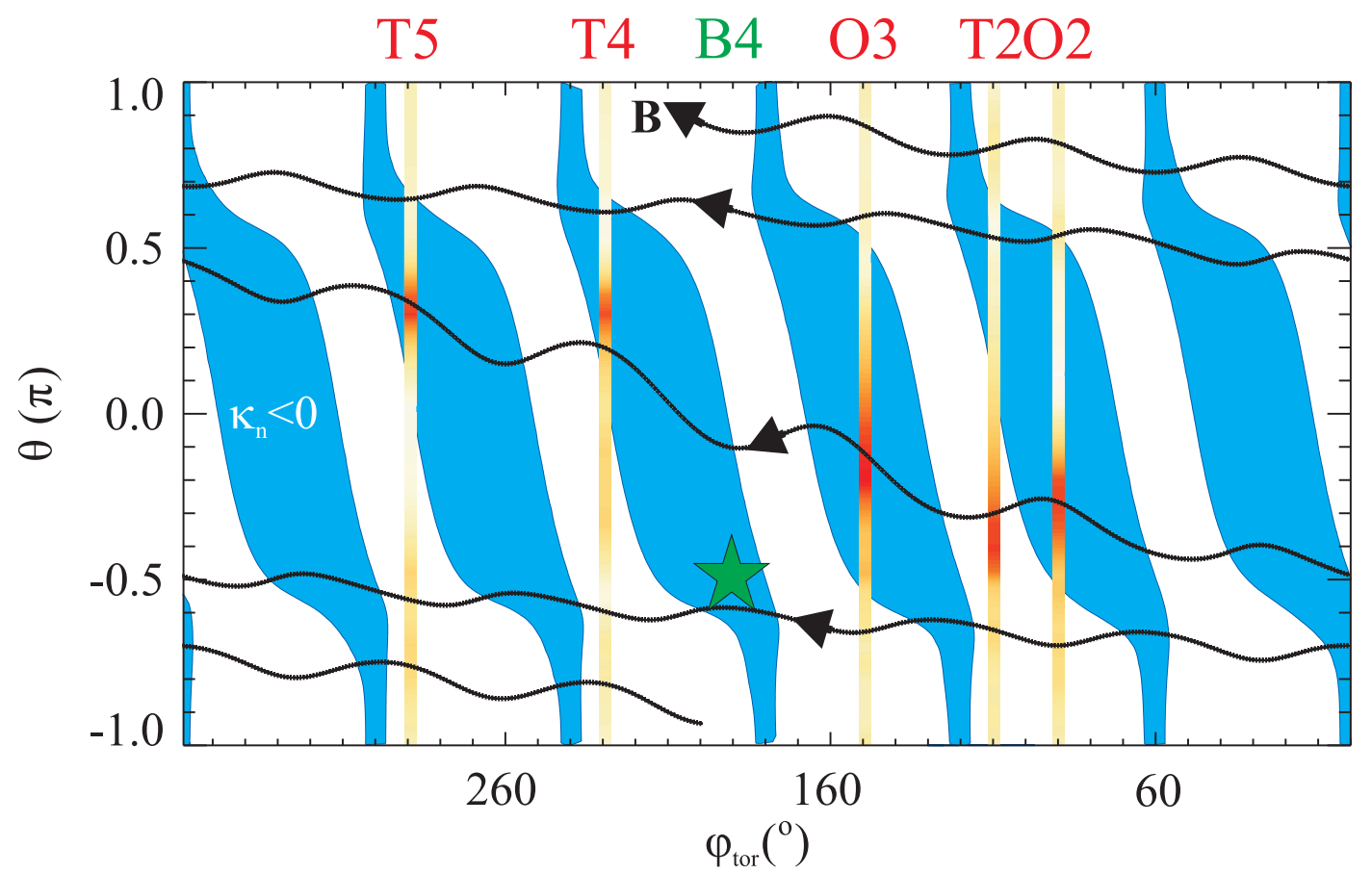

Figure 12. Poloidal turbulent transport profiles (red corresponds to high transport) measured at different toroidal positions on a flux surface at reversed field in the same representation as in Fig. 11.

to a parallel extended drive of instability. It seems that a single and most unstable mode exists which is parallel extended summing up the geometry drive of unfavorable regions on the flux surface. In addition, the transport maxima are not found in regions of high local magnetic shear at the top and the bottom of the flux surface (cp. Fig. 2). This suggests the stabilizing role of local magnetic shear described in Refs. [15, 14].

Comparing Fig. 4, bottom, with Fig. 11 reveals that the parallel extension of the region of maximum transport is about one toroidal turn $l_{\text {inst }} \approx 3.8 \mathrm{~m}$. Therefore, it is much shorter than the parallel extension of the coherent modes of $l_{\|, 0 \%} \approx 18 \mathrm{~m}$. Hence, the poloidal $m=4$ structure found in the correlation analysis is not evident in the transport measurements. In addition, neither a toroidal $n=6$ structure according to the symmetry of the device nor any other parallel overlapping of different unstable regions is observed. This leads to the conclusion that the turbulent transport drive lacks in toroidal symmetry.

Conceivably, a relatively larger population of toroidally trapped electrons (cp. Fig. 2, a) associated with the heating scheme at the upper hybrid [55] could drive the drift waves locally unstable, and hence break the toroidal symmetry. Indeed, an additional local turbulent transport maximum is found at port $\mathrm{O} 3$ when the toroidal position of the launching antenna of the microwave is changed from port B4 to port O5. The position of the main transport maximum, however, is the same in both cases. This points to a dominant influence of the magnetic field configuration on turbulent transport which is kept fixed in this investigation. Here, magnetic error fields due to 
a coil displacement as they were found in flux-surface measurements [60] break the toroidal symmetry possibly fostering a single localized unstable mode in a particular toroidal region of the torus.

In summary, turbulent transport is found at the outboard side in regions of unfavorable curvature. The area of maximum transport is extended along a field line for about $l_{\text {inst }} \approx 3.8 \mathrm{~m}$, and shifts poloidally when the magnetic field direction is reversed.

\section{SUMMARY AND CONCLUSION}

As theoretical works have shown, local magnetic field parameters strongly modify turbulence properties in the edge of fusion plasmas. The measurements reported here also point to local effects of the magnetic field geometry on turbulence confirming the theoretical outcomes. In dedicated experiments carried out in the stellarator configuration of TJ-K in unprecedented detail, locally reduced poloidal correlation lengths are found in regions of high absolute local magnetic shear $|S|$ pointing to a tilting of turbulent eddies by the magnetic field structure. Furthermore, a local influence of unfavourable curvature is suggested by the fact that the turbulent transport measured at 160 positions on a single flux surface peaks in regions of negative normal curvature $\kappa_{n}$. These locations of transport maxima depend on the magnetic field-direction which is possibly connected to the influence of the geodesic curvature $\kappa_{g}$.

The measured values of parallel correlation lengths and the propagation velocities of turbulent structures are in agreement with the expectations for drift-wave turbulence. However, the poloidal dependencies of these quantities show surprising sign reversals at the top and bottom of the flux surface which are sensitive to the magnetic field direction. Despite this complex dynamics, the poloidal displacement of turbulent structures with respect to the field line is quantitatively covered by a simple model. It assumes the creation of perfectly field aligned turbulent structures that propagate radially outward to a flux surface with another rotational transform.

The measurements also raise questions e.g. concerning the origin of the complex propagation pattern of turbulent structures. Therefore, dedicated measurements incorporating radial structure sizes and velocities are left for the future. But also quantitative comparisons with numerical turbulence models are envisaged in order to better interpret the findings.

Since the experimental results reported here qualitatively support the theoretical outcomes, the promising approach of confinement optimization by shaping of the magnetic field should vigorously extended based on existing models.

\section{References}

[1] B. Carreras, Plasma Science, IEEE Transactions on 25, 1281 (1997).

[2] W. Horton, Rev. Mod. Phys. 71, 735 (1999).

[3] F. Wagner, Plasma Physics and Controlled Fusion 49, B1 (2007).

[4] H. E. Mynick, P. Xanthopoulos, and A. H. Boozer, Physics of Plasmas 16, 110702 (2009). 
[5] A. Hasegawa and M. Wakatani, Phys. Rev. Lett. 50, 682 (1983).

[6] A. Bhattacharjee, J. E. Sedlak, P. L. Similon, and M. N. Rosenbluth, Phys. Fluids 26, 880 (1983).

[7] R. E. Waltz and A. H. Boozer, Phys. Fluids B5, 2201 (1993).

[8] A. Kendl and H. Wobig, Phys. Plasmas 6, 4714 (1999).

[9] M. Persson, M. Nadeem, J. L. V. Lewandowski, and H. J. Gardner, Plasma Phys. Controll. Fusion 41, 203 (1999).

[10] M. Nadeem, T. Rafiq, and M. Persson, Phys. Plasmas 8, 4375 (2001).

[11] M. H. Nasim, T. Rafiq, and M. Persson, Plasma Phys. Controll. Fusion 46, 193 (2004).

[12] R. Kleiber, Physics of Plasmas 8, 4090 (2001).

[13] G. Jost, T. M. Tran, W. A. Cooper, L. Villard, and K. Appert, Phys. Plasmas 8, 3321 (2001).

[14] T. Rafiq, R. Kleiber, M. Nadeem, and M. Persson, Physics of Plasmas 9, 4929 (2002).

[15] V. Kornilov, R. Kleiber, R. Hatzky, L. Villard, and G. Jost, Phys. Plasmas 11, 3196 (2004).

[16] B. Scott, Phys. Lett. A 320, 53 (2003).

[17] R. Kleiber and B. Scott, Phys. Plasmas 12, 102507 (2005).

[18] A. Kendl, J. Plasma Phys. 72, 1145 (2006).

[19] P. Xanthopoulos, F. Merz, T. Grler, and F. Jenko, Phys. Rev. Lett. 99, 035002 (2007).

[20] T.-H. Watanabe, H. Sugama, and S. Ferrando-Margalet, Phys. Rev. Lett. 100, 195002 (2008).

[21] J. A. Baumgaertel, E. A. Belli, W. Dorland, W. Guttenfelder, G. W. Hammett, D. R. Mikkelsen, G. Rewoldt, W. M. Tang, and P. Xanthopoulos, Phys. Plasmas 18, 122301 (2011).

[22] H. E. Mynick, N. Pomphrey, and P. Xanthopoulos, Phys. Plasmas 18, 056101 (2011).

[23] T. Crowley and E. Mazzucato, Nucl. Fusion 25, 507 (1985).

[24] T. Rhodes, R. Taylor, E. Doyle, J. N. Luhmann, and W. Peebles, Nuclear Fusion 33, 1787 (1993).

[25] D. L. Brower, W. A. Peebles, and N. C. Luhmann Jr., Nucl. Fusion 27, 2055 (1987).

[26] A. Wan, B. LaBombard, B. Lipschultz, and T. Yang, Journal of Nuclear Materials 145-147, 191 (1987).

[27] G. Tynan, J. Boedo, D. Gray, R. V. Nieuwenhove, G. V. Oost, and R. Weynants, Journal of Nuclear Materials 196-198, 770 (1992).

[28] C. Watts, R. F. Gandy, G. Cima, R. V. Bravenec, D. W. Ross, A. J. Wootton, A. Ouroua, J. W. Heard, T. P. Crowley, P. M. Schoch, D. L. Brower, Y. Jiang, B. Deng, C. W. Domier, and J. N. C. Luhmann, Physics of Plasmas 3, 2013 (1996).

[29] G. Kirnev, V. Budaev, S. Grashin, L. Khimchenko, and D. Sarytchev, Nuclear Fusion 45, 459 (2005).

[30] N. Fedorczak, J. Gunn, P. Ghendrih, P. Monier-Garbet, and A. Pocheau, Journal of Nuclear Materials 390-391, 368 (2009).

[31] C. Silva, H. Figueiredo, P. Duarte, and H. Fernandes, Plasma Physics and Controlled Fusion 53, 085021 (2011).

[32] M. Ramisch, N. Mahdizadeh, A. Köhn, P. Manz, and U. Stroth, Proc. of the $35^{\text {th }}$ EPS Conference on Plasma Physics, Hersonissos, Crete (The European Physical Society, Hersonissos, 2008), pp. $1-4$.

[33] G. Birkenmeier, M. Ramisch, P. Manz, B. Nold, and U. Stroth, Phys. Rev. Lett. 107, 025001 (2011).

[34] M. Ramisch, E. Häberle, N. Mahdizadeh, and U. Stroth, Plasma Sources Sci. Technol. 17, 024007 (2008).

[35] C. Lechte, S. Niedner, and U. Stroth, New J. Phys. 4, 34.1 (2002).

[36] M. Ramisch, N. Mahdizadeh, U. Stroth, F. Greiner, C. Lechte, and K. Rahbarnia, Phys. Plasmas 12, 032504 (2005).

[37] M. Ramisch, F. Greiner, N. Mahdizadeh, K. Rahbarnia, and U. Stroth, Plasma Phys. Controll. Fusion 49, 777 (2007).

[38] T. Happel, F. Greiner, N. Mahdizadeh, B. Nold, M. Ramisch, and U. Stroth, Phys. Rev. Lett. 102, 255001 (2009).

[39] J. Bleuel, M. Endler, H. Niedermeyer, M. Schubert, H. Thomsen, and The W7-AS Team, New J. 
Phys. 4, 38.1 (2002).

[40] S. Marsen, M. Otte, and F. Wagner, Contributions to Plasma Physics 48, 467 (2008).

[41] N. Mahdizadeh, F. Greiner, T. Happel, A. Kendl, M. Ramisch, B. D. Scott, and U. Stroth, Plasma Phys. Controll. Fusion 49, 1005 (2007).

[42] N. Krause, C. Lechte, J. Stöber, U. Stroth, E. Ascasibar, J. Alonso, and S. Niedner, Rev. Sci. Instrum. 73, 3474 (2002).

[43] S. Enge, G. Birkenmeier, P. Manz, M. Ramisch, and U. Stroth, Phys. Rev. Lett. 105, 175004 (2010).

[44] U. Stroth, F. Greiner, C. Lechte, N. Mahdizadeh, K. Rahbarnia, and M. Ramisch, Phys. Plasmas 11, 2558 (2004).

[45] S. Niedner, B. D. Scott, and U. Stroth, Plasma Phys. Controll. Fusion 44, 397 (2002).

[46] K. Rahbarnia, E. Holzhauer, N. Mahdizadeh, M. Ramisch, and U. Stroth, Plasma Phys. Controll. Fusion 50, 085008 (2008).

[47] P. Manz, M. Ramisch, and U. Stroth, Plasma Phys. Controll. Fusion 51, 035008 (2009).

[48] P. Manz, M. Ramisch, and U. Stroth, Phys. Plasmas 16, 042309 (2009).

[49] P. Manz, M. Ramisch, and U. Stroth, Phys. Rev. Lett. 103, 165004 (2009).

[50] P. Manz, M. Ramisch, and U. Stroth, Phys. Rev. E 82, 056403 (2010).

[51] M. Ramisch, P. Manz, U. Stroth, G. Birkenmeier, S. Enge, E. Holzhauer, A. Köhn, and B. Nold, Plasma Physics and Controlled Fusion 52, 124015 (2010).

[52] E. Ascasibar, J. Quin, A. Fraguas, I. Pastor, and J. Herranz, Nucl. Fusion 37, 851 (1997).

[53] B. Kadomtsev and O. Pogutse, Nuclear Fusion 11, 67 (1971).

[54] J. L. Johnson and J. M. Greene, Plasma Physics 9, 611 (1967).

[55] A. Köhn, G. Birkenmeier, E. Holzhauer, M. Ramisch, and U. Stroth, Plasma Phys. Controll. Fusion 52, 035003 (2010).

[56] N. Mahdizadeh, F. Greiner, M. Ramisch, U. Stroth, W. Guttenfelder, C. Lechte, and K. Rahbarnia, Plasma Phys. Controll. Fusion 47, 569 (2005).

[57] M. Ramisch, G. Birkenmeier, A. Köhn, and U. Stroth, Proc. of the $38^{\text {th }}$ EPS Conference on Plasma Physics, Strasbourg, France (The European Physical Society, Strasbourg, 2011), pp. 1-4.

[58] N. Winsor, J. L. Johnson, and J. M. Dawson, Phys. Fluids 11, 2448 (1968).

[59] H. Wobig, Plasma Physics and Controlled Fusion 41, A159 (1999).

[60] E. Ascasibar, C. Ochando, M. A. Alejaldre, J. Alonso, R. Balbin, et al., Plasma Physics and Controlled Fusion Research (Proc. 16 ${ }^{\text {th }}$ Int. Conf., Montreal, 1996), IAEA, Vienna (IAEA, Vienna, 1996), p. 183. 